\title{
An Exponential Distribution-based Model for Equipment Remaining Life Prediction
}

\author{
MENG Xiao-Hong \\ 503 Section of Xi' an High-tech \\ Institute, 710025, \\ Hongqing Town, Xi' an, China
}

\author{
WANG Wei \\ 503 Section of Xi' an High-tech \\ Institute, 710025 , \\ Hongqing Town, Xi' an, China
}

\author{
ZHOU Yong-Tao \\ 503 Section of Xi' an High-tech \\ Institute, 710025 , \\ Hongqing Town, Xi' an, China
}

\begin{abstract}
The article proposed and established an exponential distribution-based model for equipment remaining life prediction. The model is a general decision model for detectable continuous degradation process. The modeling method and application example is introduced. Compared with other models, the model has the basic characteristics of simple and practical. It has important practice value for maintenance decision of equipment parts fault caused by gradual loss.
\end{abstract}

Keywords-Exponential distribution; remaining life; equipment maintenance

The equipment remaining life is a length of time that product from the time of starting check to the time of going wrong. Equipment failure means a state that the system or equipment and product can not execute stated function at stated condition $^{11]}$. This state can be called potential failure when the system or equipment and product are about to loss function. The components will be frayed, tired, ablated, aging and maladjusted when it was used and most of them will change from the potential failure to function failure, but it is not all the product have potential failure. For example, a certain electrical components and integrated blocks may have not any omen before happening function failure. So it is the hot topic recent years that monitoring the equipment possessed the characteristic of potential failure so as to confirm its potential failure status and forecasting the using life and remaining life of equipment to confirm the most economical maintenance time, which has significant meaning of improving the using efficiency and maintenance benefit of equipment.

I. The basic condition of equipment remaining life prediction model

The precondition of forecasting equipment remaining life is that the degeneration of equipment product function can be monitored, and can confirm a distinct potential failure status. Meanwhile, it should has enough length between the monitoring point of potential failure and happening point of function failure so as to adopt maintenance measure and prevent the happening of failure. The function degeneration of many products is a gradual process that can be described as a curve from starting degeneration to happening function failure ${ }^{[8]}$. See Fig. 1.

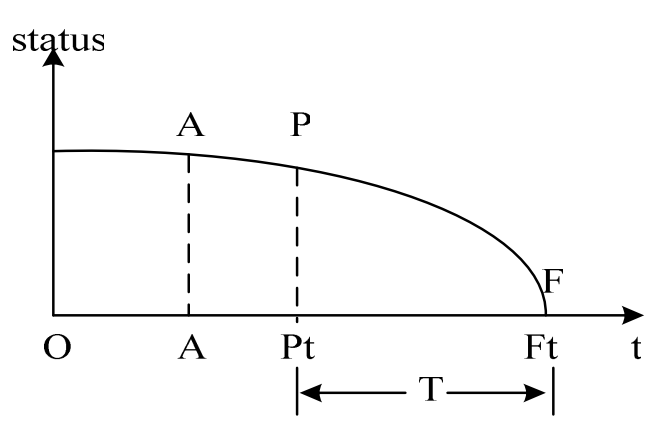

Fig. 1 potential failure to function failure

A — staring point of function degeneration; $\mathrm{P}$ - detecting point of potential failure; $\mathrm{F}$-staring point of function failure; $\mathrm{T}$-continue using time.

It can be seen form the Fig. 1 that the point $\mathrm{P}$ is the identified status of function degeneration. In other words, it is the potential failure point that is about to occur function failure. The point $\mathrm{F}$ is function failure point. There is an interval between potential failure point $\mathrm{P}$ and function failure point $\mathrm{F}$, which reflects that if there is no detection and measure, the product from starting degradation to potential failure point $\mathrm{P}$ that the failure can be detected will continue degradation until arriving at function failure point F. It is thus clear that maintaining the product at the potential failure stage of $\mathrm{P}-\mathrm{F}$ can prevent from the happening of function failure. So it is an economical maintenance manner that the product can be used at all times until arriving at the potential failure stage of $\mathrm{P}-\mathrm{F}$ that is about to occur function failure. It can also utilize all the available life of product effectively.

II. A model of equipment remaining life prediction based on exponential distribution

The paper discussed building commonly decision model that aims at detecting and continuing degeneration process.

If:

(1) The function degeneration of product or component starts from 0 . The time of status detection is discrete. The status information can be measured.

(2) The life time of product or component start from 0 (starting using) to $t_{0}$ (happening time of potential 
failure) can be called the first step, and from $t_{0}$ to $t_{j}$ (happening time of function failure) can be called the second step.

(3) The second step of the life time of product or component is delay time that related with the status information $\eta$ detected.

(4) In a certain section of delay time step $\left(t_{0}, t_{j}\right)$, a stochastic vector is obtained. Its element submits to exponential distribution. The distribution function is the function of product remaining life at $t_{i}$. (see Fig. 2)

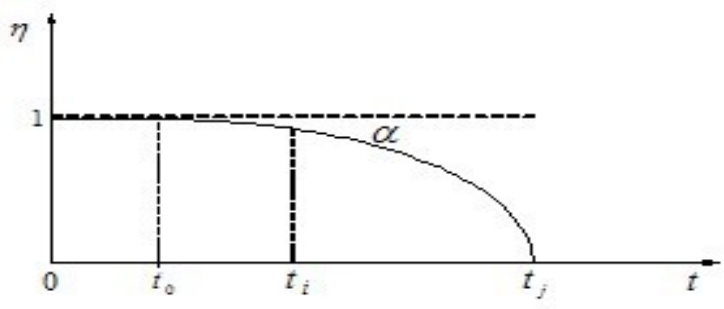

Fig. 2 The sketch map of equipment remaining life

The exponential distribution is the most basic and common failure distribution, and its distribution curve as Fig. 3.

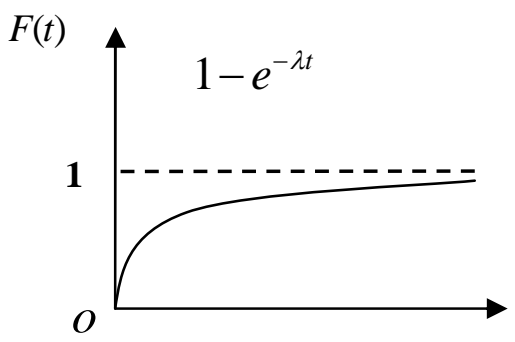

Fig. 3 The curve chart of equipment failure exponential distribution

The mathematical expression of Fig. 3 is: $F(t)=1-e^{-\lambda t}$

Transform the expression as above so as to accord with the Fig. 2:

$$
F(t)=1-e^{-\lambda t}-1
$$

$\eta$ is ordinate that represents the equipment status information.

$$
\begin{aligned}
& \eta=-e^{-\lambda t} \\
& \eta=-e^{-\lambda\left(t_{j}-t_{0}\right)} \quad\left(\lambda>0, t=t_{j}-t_{0}<0\right)
\end{aligned}
$$

In multimode:

$$
\eta=-e^{-\lambda\left(t_{j}-t_{0}\right)}+\alpha
$$

In formula, $\eta$-equipment status information.

$t_{0}$ — the time of happening equipment potential failure.

The detection point of equipment potential failure information is $t_{i}$.so the formula as above also can be written as:

$\eta=-e^{-\lambda\left(t_{j}-t_{i}\right)}+\alpha$
$\left(\lambda>0, t=t_{j}-t_{i}<0\right)$

Make: $t_{j}-t_{i}=T, T$ is equipment remaining life. Both side of the formula take the logarithm after transplant $\alpha$.

$$
\alpha-\eta=e^{-\lambda T}
$$

Because the equipment remaining life will reduce gradually in the process of using, that is to say, the time variable $T$ itself is a negative value.

$$
\begin{aligned}
& t_{j}-t_{i}<0=-T, \text { so the formula (4) can convert to: } \\
& T=\frac{\ln (\alpha-\eta)}{\lambda}
\end{aligned}
$$

The remaining life $T$ can be calculated by the formula (5) in multimode.

In formula: $\eta$-equipment status information.

$\lambda$-failure rate.

$\alpha$-shape parameter of equipment status changing curve.

$T$-equipment remaining life.

$\alpha$ is empirical coefficient, and can be got by the manner of drag-in test and modifying the constant and variable of parameter. The different equipment has different remaining life when $\alpha$ is different.

III. Applying for model of equipment remaining life prediction based on exponential distribution

Parts of data are extracted from the status monitoring data of certain parts of certain equipment engine. (The failure distribution of these parts submits to exponential distribution.) Table 1 is the result that calculated by the formula (5), and a set of curve are shown as Fig. 4.

Table 1 The failure data and remaining life of some kind of parts

\begin{tabular}{c|c|c|c}
\hline Part & $\lambda /(1 / \mathrm{h})$ & $\ln (\alpha-\eta)$ & $\mathrm{T} /(\mathrm{h})$ \\
\hline 1 & $1 / 2000$ & 0.25 & 500 \\
\hline 2 & $1 / 2700$ & 0.22 & 594 \\
\hline 3 & $1 / 1850$ & 0.43 & 795.5 \\
\hline 4 & $1 / 1000$ & 1.24 & 1240 \\
\hline
\end{tabular}




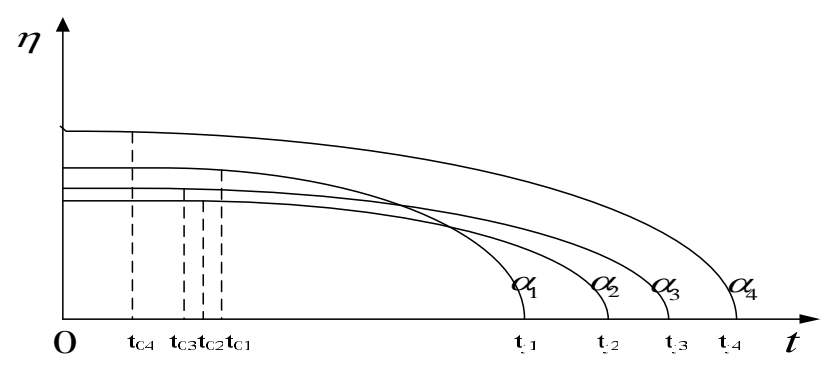

Fig. 4 The sketch map of multimode equipment remaining life

According to the formula (5), it can be seen from Fig. 3 that the shape of curve is decided by $\alpha$. The curve is steeper; the remaining life of the parts is shorter. So $\alpha$ should be called "maintenance coefficient". The remaining life of the parts is not decided by failure rate $\lambda$, and decided by $\lambda$ and the status of the parts together, and related with the shape of parts status changing curve, that is to say, related with maintenance coefficient $\alpha$. The different parts, using environment and operating person conduce the different parts status changing. When $\alpha$ is confirmed, the remaining life $T$ of the parts can be calculated by analyzing monitoring data of parts status. The higher the value of $T$ is, the longer the cycle of preventive maintenance. The lower the value of $T$ is, the shorter the cycle of preventive maintenance. The best maintenance benefit can be got by choosing advisable time to make preventive maintenance within the preventive maintenance cycle.

The parts having invariable failure rate commonly have no complex system. The product that maintained timely before occurring wear failure, the parts failure conduced by stochastic high stress and the parts that the failure is weak wear type occurred within using life, whose failure all submit to exponential distribution. The most representative failure is the failure caused by engine damage. It has good effect that checking many equipment parts based on exponential distribution function, but exponential distribution go against with finding equipment early failure.

\section{References}

[1] Zhaoshun Sheng, Qiling Yin. Applying for Equipment Status Monitoring and Failure Diagnoses Technology [M]. Beijing: Chemical Engineering Press. 2003, 2.

[2] Bibo Zhang, Wenlong Cong. Equipment Status Monitoring and Failure Diagnoses [M]. Beijing: Chemical and Industry Press. 2005, 4.

[3] Daqi Zhu. Method of Failure Diagnoses Knowledge-based [J]. Anhui Industry College Journal, 2002, 19(3): 197-204.

[4] Qianxiang Zhou, Hualing Guo. Failure Characteristic of Manned Spacecraft and Research of Diagnoses Technology [J]. China Spaceflight, 1999(9): 30-32.

[5] Erik, O. Fault Diagnosis in Industry Using Sensor Readings and Case-Based Reasoning[J].Journal of Intelligent \& Fuzzy Systems. 2004, Vol. 5: 41-46.

[6] BOCANIALA D, COSTAB J s D. Application of novel fuzzy classifier to fault detection and isolation of the DAMADICS benchmark problem Cosmin[J]. Control Engineering Practice 。 2006(14): 653-669.

[7] Jun Yang, Yu Zhao, Xuejing Li, Dan Yu. Comprehensive Assess Method of Complex System Average Remaining Life [J]. Spaceflight Journal, 2007(11): 28.

[8] Kejun Xu. Space Launch Failure Diagnoses Technology [M]. Beijing: National Defense Engineering Press, 2007: 51. 